\title{
Social behavior following traumatic brain injury and its association with emotion recognition, understanding of intentions, and cognitive flexibility
}

\author{
MAARTEN MILDERS, ${ }^{1}$ MAGDALENA IETSWAART, ${ }^{2}$ JOHN R. CRAWFORD, ${ }^{1}$ \\ AND DAVID CURRIE ${ }^{3}$ \\ ${ }^{1}$ School of Psychology, University of Aberdeen, Aberdeen, United Kingdom \\ ${ }^{2}$ School of Psychology and Sport Sciences, University of Northumbria, Newcastle upon Tyne, United Kingdom \\ ${ }^{3}$ Department of Neurosurgery, Aberdeen Royal Infirmary, Aberdeen, United Kingdom
}

(Received August 17, 2007; Final Revision November 8, 2007; AccePted November 8, 2007)

\begin{abstract}
Although the adverse consequences of changes in social behavior following traumatic brain injury (TBI) are well documented, relatively little is known about possible underlying neuropsychological deficits. Following a model originally developed for social behavior deficits in schizophrenia, we investigated whether impairments in emotion recognition, understanding of other people's intentions ("theory of mind"), and cognitive flexibility soon after first TBI or 1 year later were associated with self and proxy ratings of behavior following TBI. Each of the three functions was assessed with two separate tests, and ratings of behavior were collected on three questionnaires. Patients with TBI ( $n=33$ ) were impaired in emotion recognition, "theory of mind," and cognitive flexibility compared with matched orthopedic controls $(n=34)$. Proxy ratings showed increases in behavioral problems 1 year following injury in the TBI group but not in the control group. However, test performance was not associated with questionnaire data. Severity of the impairments in emotion recognition, understanding intention, and flexibility were unrelated to the severity of behavioral problems following TBI. These findings failed to confirm the used model for social behavior deficits and may cast doubt on the alleged link between deficits in emotion recognition or theory of mind and social functioning. (JINS, 2008, 14, 318-326.)
\end{abstract}

Keywords: Traumatic brain injury, Social behavior, Emotion recognition, Theory of mind, Cognitive flexibility, Follow-up

\section{INTRODUCTION}

Changes in emotional and social behavior after traumatic brain injury (TBI), as expressed in emotional lability, indifference to other people's feeling, poor social judgment and communication, or impulsivity, are relatively common and can have serious consequences for psychosocial outcome (Kendall \& Terry, 1996; Levin, 1995; Morton \& Wehman, 1995). Survivors of TBI may fail to return to work or to maintain meaningful social relationships as a result of these changes (Brooks et al., 1987; Kendall \& Terry, 1996, Malia et al., 1995; Ownsworth \& McKenna, 2004). For relatives

Correspondence and reprint requests to: Maarten Milders, School of Psychology, University of Aberdeen, Aberdeen AB24 2UB, United Kingdom. E-mail m.milders@abdn.ac.uk of patients with TBI, the behavioral changes can be a greater burden than the physical or cognitive impairments (Brooks et al., 1986; Kinsella et al., 1991), even many years after the injury (Koskinen, 1998). Although the adverse consequences of changes in emotional and social behavior have been well documented, relatively little is known about the impairments that may underlie these changes.

Models of psychosocial outcome following TBI include neuropsychological deficits as factors directly contributing to postinjury behavior (Kendall \& Terry, 1996; Prigatano, 1999). Previous outcome studies in TBI tended to focus on cognitive impairments in memory, processing speed, or attention as possible predictors, but their contribution was often limited (Bowman, 1996; Ownsworth \& McKenna, 2004; Vilkki et al., 1994). Relatively few studies have examined the association between postinjury behavior and 
impairments in functions that are generally regarded as important for adequate social behavior, namely recognizing other people's emotions or intentions or understanding of social situations. The aim of the current study was to investigate the relation between these functions and social behavior following TBI. As framework, we used a model proposed by Corrigan (1997) to account for inadequate social behavior in patients with schizophrenia. The model proposes three main stages involved in social functioning: perception of social cues, retrieval of social knowledge, and response selection. Impairments at each of these stages could result in maladaptive behavior. Applying this model to patients with TBI suggests that problems in social behavior could result from (1) insensitivity to important social cues, such as emotional expressions; (2) impaired understanding of social situations and other people's intentions; (3) failures to adjust one's behavior in accordance with the demands of the situation, that is, in flexibility.

Impaired recognition of emotions as expressed in the face, the voice, or body posture has been reported in patients with TBI (Braun et al., 1989; Croker \& McDonald, 2005; Green et al., 2004; Hopkins et al., 2002; Jackson \& Moffat, 1987; McDonald \& Flanagan, 2004; McDonald \& Saunders, 2005; Milders et al., 2003; Pettersen, 1991; Spell \& Frank, 2000). Jackson and Moffat (1987) already speculated that the impairment in recognizing emotional expressions could play a role in the inadequate social behavior following TBI, without actually investigating that possibility. Several studies have reported on the relationship between emotion recognition and behavior following TBI. Pettersen (1991) noted that head injured children who were impaired at recognizing facial expressions were also rated by their parents as showing less socially appropriate behavior (e.g., failing to respond to indirect cues or to apologize for hurting someone's feelings). Croker and McDonald (2005) reported a relationship between facial expression recognition and changes in subjective experience of emotion following TBI. Poor matching of facial expressions was associated with reported reductions in the experiences of sadness and fear. A similar association between the ability to recognize emotional expressions and subjective emotional experience was found by Hornak et al. (1996) in patients with frontal lesions due to various etiologies, including TBI. Furthermore, patients from the same sample who were rated as poor at interpreting other people's moods by nursing staff also performed poorly on a test of emotion recognition (Hornak et al., 1996).

A second function to contribute to effective social behavior is the ability to infer and understand other people's intentions and beliefs, also referred to as Theory of Mind (ToM, Channon \& Crawford, 2000; Happé et al., 2001). Impaired performance in ToM tests has been reported in patients with TBI (Bibby \& McDonald, 2005; Channon \& Crawford, 2000; Channon et al., 2005; McDonald \& Flanagan, 2004; Milders et al., 2003, 2006). Although few studies investigated the association between impairments in ToM and behavioral changes following brain injury, there are indications supporting such a relationship. Gregory et al. (2002) reported an association between ToM impairments and maladaptive social and emotional behavior in patients with frontotemporal dementia, while in an earlier study we found a similar association in patients with severe TBI (Milders et al., 2003). Cicerone and Tanenbaum (1997) also described impaired performance on tests assessing interpretation of social situations in a patient with emotional and social behavior problems following TBI.

The third stage in Corrigan's model is response selection. Impairments in response selection could mean that patients are unable to adjust their behavior to the demands of the situation. Flexible response selection is an example of executive functioning. Impairments in executive functioning, as reflected in inflexibility and impulsivity, are frequently reported following TBI (Levin, 1995; Tate, 1999). Reduced flexibility in TBI patients could adversely affect their ability to cope with environmental demands (Prigatano, 1992) or to participate in adaptive social communication (Godfrey \& Shum, 2000). Consistent with these suggestions, Vilkki et al. (1994) and Nybo et al. (2004) showed that TBI patients' performance on tests of cognitive flexibility predicted their ability to return to work and their level of social activity several months later.

In summary, there is evidence in patients with TBI for impairments in those functions that, in Corrigan's (1997) model, are proposed as prerequisites for adequate social behavior, namely emotion recognition, understanding of social situations and other people's intentions, and cognitive flexibility. Although there have been attempts to relate impairments in each of these functions individually to behavior following TBI, few studies incorporated all three functions to investigate their relative contribution to postinjury behavior. For the study reported here, we assessed emotion recognition, ToM, and cognitive flexibility and related test performance to self and proxy ratings of postinjury behavior in a consecutive sample of patients with TBI. Patients with TBI and orthopedic controls were assessed shortly after injury and at 1-year follow-up. The main question was whether impairments in emotion recognition, ToM, and cognitive flexibility were associated with social behavior 1 year following TBI. Significant associations between test performance and ratings of behavior could point at deficits underlying changes in social behavior. In addition, the longitudinal design allows us to examine whether impairments shortly after injury might serve as predictors of behavior 1 year later.

\section{METHOD}

\section{Participants}

Patients with first incidence TBI were recruited consecutively from the Department of Neurosurgery at Aberdeen Royal Infirmary and orthopedic control participants were recruited from the Orthopedic Trauma Unit of the same 
hospital to match the TBI group for gender, age, years of education, and socioeconomic status. Exclusion criteria were a neurological or psychiatric history, dementia, or a history of alcohol or drug dependency. All participants were screened for language comprehension deficits using the Complex Ideation subtest of the Boston Diagnostic Aphasia Examination (Goodglass \& Kaplan, 1983), which resulted in exclusion of one patient with TBI.

The TBI group that entered the study consisted of 33 patients (28 males, 5 females). The control group consisted of 30 males and 4 females. The TBI and control groups were matched for age (TBI: $M=37.5, S D=16.1$ years; control: $M=35.6, S D=13.1$ years), years of education (TBI: $M=13.1, S D=2.1$; control: $M=13.5, S D=2.2$ ), and socioeconomic status, as based on the United Kingdom's National Statistics Social Economic Classification (Office of National Statistics, 2005) (TBI: $M=4.3, S D=1.9$; control: $M=3.8, S D=2.1$ ). The two groups were comparable in that all participants had sustained traumatic injuries, which either included head injuries or were restricted to orthopedic injuries. A relative or significant other of each participant was recruited to provide proxy ratings on premorbid and postinjury behavior.

The average lowest Glasgow Coma Scale (GCS) score in the TBI group was 10 (range, 3-15), and mean duration of posttraumatic amnesia (PTA) was 12.5 days (range, 1-90 days). Following a conventional classification (Teasdale \& Jennett, 1974), 9 patients were classed as mild (GCS $13-15$ or PTA $<24 \mathrm{hr}$ ), 14 as moderate (GCS 9-12 or PTA $1-7$ days), and 10 as severe (GCS $<9$ or PTA $>$ 7 days) TBI. All participants and their relatives gave informed consent to participate in the study, which had been approved by the Grampian Research Ethics Committee.

\section{Measures}

To avoid equating functions or behavior with the instrument used, all variables of interest were assessed with at least two independent measures, which are described below.

\section{Questionnaires}

Neuropsychology Behavior and Affect Profile (NBAP; Nelson et al., 1998). The NBAP is a 106-item questionnaire specifically designed to assess the emotional and behavioral consequences of acquired brain damage. The Self version was completed by patients and controls and the Observer version by a relative or significant other. Each item is rated in relation to premorbid and postinjury behavior as "agree," meaning typically or often, or "disagree," meaning seldom or hardly at all. "Agree" is scored as 1 and "disagree" as 0 . Item scores are allocated to one of five subscales (Indifference, Inappropriateness, Pragnosia, Depression, Mania) and are summed into a Total NBAP score. Higher scores indicate more behavioral problems.

Katz Adjustment Scale-Revised (KAS-R: Goran \& Fabiano, 1993). This 79-item questionnaire is an adaptation of the Katz Adjustment Scale to assess behavioral functioning following TBI. The items relate to emotional and behavioral characteristics and are rated by a significant other of the patient on a 4-point scale (1, almost never; 4, almost always). Ratings on each item are summed into a Total score and can be allocated to one of 10 subscales (belligerence, apathy, social irresponsibility, orientation, antisocial behavior, speech/cognitive dysfunction, bizarre acts or beliefs, paranoid ideas, verbal expansiveness, emotional sensitivity). Higher scores represent more severe behavioral problems.

Dysexecutive Questionnaire (DEX: Wilson et al., 1996). This instrument is part of the Behavioral Assessment of Dysexecutive Syndrome battery. The 20 items refer to behavioral and emotional characteristics and are rated on a 5-point scale ( 0 , never; 4 , always). Ratings are summed into a Total DEX score and can be allocated to Behavior, Cognition, and Emotion subscores. Higher scores on the DEX indicate more behavioral problems.

A further questionnaire was included to estimate the subjective levels of distress in the participants and their relatives. The Hospital Anxiety and Depression Scale (HADS; Zigmond \& Snaith, 1983) is a self-report measure of anxiety and depression. The 14 items are rated on a 4-point scale and the higher the total score, the higher the level of distress. Proxy ratings on the HADS related to the relatives' own levels of distress rather than those of the patients or controls.

\section{Emotion Recognition}

\section{Recognizing facial expressions}

This test consists of 60 photographs from a standard set of facial expressions (Ekman \& Friesen, 1976). The expressions depicted are fear, disgust, anger, happiness, sadness, or surprise, each displayed by 10 different individuals. The photographs are presented one by one and the names of the six emotions are printed next to each photograph. The task is to choose the emotion name that best described the facial expression shown.

\section{Recognizing emotions in the voice}

Recognition of emotional prosody was tested with a subtest from the Florida Affect Battery (FAB: Bowers et al., 1998). Twenty neutral sentences are spoken in one of five possible tones of voice: happy, sad, angry, fearful, neutral. The five possible emotion labels are presented to participants, who choose for each sentence the emotion label that best describes the affective prosody.

\section{Theory of Mind}

Faux Pas Test (Stone et al., 1998)

This test consists of 20 vignettes, 10 describe a social faux pas, and 10 contain no faux pas. Each vignette is printed on 
a sheet that is placed in front of the participant while the experimenter reads the vignette out aloud. Once the vignette is read, participants answer several questions while keeping the printed text in front of them. Following vignettes containing a faux pas, five questions assess participants' detection of the social faux pas and their understanding of the intentions and beliefs of the characters in the story. Following vignettes without faux pas, one question is asked to assess detection of the absence of a faux pas and a second question tests participants' general comprehension of the vignette. Participants receive one point for each correct response. The score referring to understanding of intentions in the 10 vignettes with faux pas was taken as the measure of performance on this task, as this score discriminated best between patients and controls (see Milders et al., 2006, for more details on the results of this task).

\section{Cartoon test (Happé et al., 1999)}

This test consists of 12 cartoons of humorous situations. In six cartoons, the joke is based on false belief of a character in the cartoons and would probe ToM ability (mental items). In the remaining six cartoons, the joke is based on a physical anomaly (physical items). Participants are presented with one cartoon at a time and asked to explain why the cartoon is funny. An answer receives three points if it provides a complete and explicit explanation, two points if the explanation is incomplete or implicit, and one point if relevant parts of the cartoon are mentioned, without further explanation. Incorrect or irrelevant answers receive no points. Scores on the mental cartoons and the physical cartoons are summed into separate subscores. Here, we report only performance on the mental cartoons. A detailed report of performance on this task can be found in Milders et al. (2006).

\section{Cognitive Flexibility}

\section{Brixton Spatial Anticipation Task (Burgess \& Shallice, 1997)}

This task consists of 56 pages each containing two rows of five circle outlines. One of the circles is filled, and the position of this filled circle varies across successive pages following predefined rules. The participants' task is to detect the rules and use these to predict the position of the filled circle on the next page. Performance is expressed as the number of correct responses.

\section{Alternating Fluency Test (Downes et al., 1993)}

This verbal fluency test requires the generation of names of exemplars from alternating categories. The test consists of three subtasks: (1) words beginning with $\mathrm{T}$ and countries; (2) words beginning with D and colors; (3) words beginning with $\mathrm{C}$ and occupations. Participants receive one point for each correct exemplar produced within $60 \mathrm{~s}$. Correct scores from the three subtasks are summed into a single Alternating Fluency score.

\section{PROCEDURE}

Patients and controls were tested individually either at their home, the School of Psychology, or on the hospital ward. All participants were tested twice on the tests mentioned above, shortly after injury and again 1 year later. The average interval between injury and first assessment was 2.1 $(S D=1.8)$ months in the TBI group and $1.2(S D=1.0)$ months in the orthopedic group; this difference was significant $(p<.05)$. The average interval between first assessment and follow-up was $11.8(S D=1.9)$ months for the TBI group and $11.4(S D=1.8)$ months for the controls; this difference was not significant. Of the 67 participants who were tested shortly following injury, 61 participants, 30 patients with TBI ( 5 female, 25 male) and 31 controls (4 female, 27 male), returned for follow-up. The groups retested at follow-up were still matched for age (TBI: $M=37.3$, $S D=16.3$ years; control: $M=36.1, S D=13.2$ years), years of education (TBI: $M=13.1, S D=2.1$; control: $M=13.7$, $S D=2.2$ ), and social economic status (TBI: $M=4.4, S D=$ 1.9; control: $M=3.7, S D=2.0$ )

The questionnaires were completed at around the same time as the tests. Proxy ratings of premorbid behavior were collected at the time of the first assessment using the Observer versions of the DEX and the KAS-R. Proxy ratings of postinjury behavior were collected at the time of the 1-year follow-up on the KAS-R, DEX, and NBAP. Because of the format of the NBAP, proxy ratings of premorbid behavior on this instrument were collected at follow-up. At followup, a relative of each participant completed the Self version of the HADS to assess their level of anxiety and depression. Self ratings of postinjury behavior were obtained at follow-up on the Self versions of the DEX and NBAP and self ratings of premorbid behavior were obtained on the NBAP. Finally, all participants completed the Self version of the HADS.

\section{RESULTS}

\section{Ratings of Premorbid and Postinjury Behavior}

\section{Neuropsychology Behavior and Affect Profile (NBAP)}

Mean ratings of premorbid and postinjury behavior on the NBAP from the patients, the controls, and their relatives are displayed in Table 1. The scores represent the number of items endorsed, and higher scores indicate more behavioral difficulties. Comparison of the NBAP Total scores between patients and controls based on proxy ratings with nonparametric Mann-Whitney $U$ tests showed no differences in premorbid behavior ( $p=.49$ ) but significantly higher ratings for the patient group at follow-up $(z=3.31 ; p<.01)$. Within group comparisons using nonparametric Wilcoxon tests showed a significant increase in proxy ratings from premorbid to postinjury for patients $(z=3.05 ; p<.01)$, but not for controls $(z<1 ; p>.6)$. Comparison of the NBAP 
Table 1. Mean ( $S D$ s) ratings of premorbid and postinjury behavior for the TBI and the control group as obtained from a relative of significant other (proxy) or from the patients themselves (self)

\begin{tabular}{|c|c|c|c|c|c|c|c|c|}
\hline & \multicolumn{2}{|c|}{ Proxy Premorbid } & \multicolumn{2}{|c|}{ Postinjury } & \multicolumn{2}{|c|}{ Self Premorbid } & \multicolumn{2}{|c|}{ Postinjury } \\
\hline & TBI & Control & TBI & Control & TBI & Control & TBI & Control \\
\hline NBAP & $8.10(7.52)$ & $6.61(5.56)$ & $14.90(10.66)$ & $6.87(4.55)$ & $11.50(8.71)$ & $13.68(9.11)$ & $16.53(11.13)$ & $15.25(10.82)$ \\
\hline DEX & $11.87(9.35)$ & $7.70(6.10)$ & $21.34(18.27)$ & $7.74(5.28)$ & & & $17.73(11.53)$ & $18.17(11.10)$ \\
\hline KAS-R & $103.67(15.43)$ & $98.27(11.98)$ & $116.37(22.66)$ & $100.78(10.56)$ & & & & \\
\hline $\mathrm{HADS}^{\mathrm{a}}$ & & & $10.76(7.20)$ & $6.27(4.58)$ & & & $10.07(7.47)$ & $7.36(5.48)$ \\
\hline
\end{tabular}

Note. $\mathrm{TBI}=$ traumatic brain injury; NBAP $=$ Neuropsychology Behavior and Affect Profile; DEX $=$ Dysexecutive Questionnaire; KAS-R $=$ Katz Adjustment Scale-Revised; HADS = Hospital Anxiety and Depression Scale.

aThe HADS proxy score reflects relatives' own levels of distress.

self ratings between patients and controls showed no differences in premorbid or postinjury ratings $(p>.27)$. Within both groups, NBAP self ratings increased significantly from premorbid to postinjury $(z \leq 2.63 ; p<.01)$. Both patients and controls reported more behavioral difficulties after than before injury. Comparison of proxy ratings on the five NBAP subscales showed no group differences in premorbid behavior. Postinjury ratings of the patients were higher than the controls' on all five subscales $(z \geq 2.2 ; p<.03)$, except Mania. Self ratings on the subscales showed no significant group differences for premorbid or postinjury behavior. The only exception was self-rated premorbid Inappropriateness, which scored higher in the controls than in the patients $(z=$ $2.89 ; p<.01)$.

\section{Katz Adjustment Scale-Revised (KAS-R)}

The KAS-R was completed by a relative or significant other only. Total KAS-R scores showed no group difference for premorbid behavior (see Table 1), but significantly higher postinjury ratings for the patient group $(z=2.51 ; p<.05)$. Within-group comparisons showed a significant increase in KAS-R ratings from premorbid to postinjury in the patient group $(z=2.75 ; p<.01)$, but not in the control group ( $p>$ $.05)$. The total KAS-R score can be subdivided into 10 subscales. Only one of the subscale premorbid ratings (belligerence) was significantly higher in patients than controls $(z=2.17 ; p<.05)$, whereas postinjury ratings on the majority of the subscales $(6 / 10)$ were significantly higher in the patient group $(z \geq 2.18 ; p<.05)$.

\section{Dysexecutive Questionnaire (DEX)}

Total DEX proxy ratings of premorbid behavior were not different in patients and controls (see Table 1), but postinjury ratings were significantly higher in the patients $(z=$ $2.76 ; p<.01)$. Proxy DEX scores increased from premorbid to postinjury behavior in the patient group $(z=2.84$; $p<.01)$, but not in the control group $(p>.4)$. Group comparisons of the DEX subscores showed no differences in proxy ratings of premorbid behavior but significantly higher proxy ratings of postinjury behavior in the patient group on all three subscores $(z \geq 2.02 ; p<.05)$. Self rat- ings of postinjury behavior either on total DEX score or individual subscores did not differ between the patients and controls. Self ratings of premorbid behavior were not collected with the DEX.

\section{Hospital Anxiety and Depression Scale (HADS)}

Self ratings of emotional distress 1 year after injury were not different between patients and controls $(p=.15)$. However, self ratings from relatives were significantly higher in the relatives of the patients with TBI $(z=2.26 ; p<.05)$, indicating more psychological distress in this group. The relatives' HADS scores also correlated with the relatives' ratings of the patients' behavior 1 year after TBI on the three questionnaires $(r>.62 ; p<.001)$. The more severe the behavioral problems in the patients with TBI, as reported by their relatives, the greater the relatives' own emotional distress.

To summarize, the results from the KAS-R, DEX, and NBAP based on proxy ratings are consistent in showing no differences in premorbid behavior between patients and controls, but significantly higher postinjury ratings for the patients. Proxy ratings of premorbid and postinjury behavior changed little in the control group but increased in the patient group, indicating an increase in behavioral difficulties 1 year after injury. On each questionnaire, results based on the total scores were mirrored in the large majority of the subscales.

In the patient group, self ratings and proxy ratings of premorbid behavior, as assessed with the NBAP, correlated significantly $(r=.51 ; p<.01)$ as did self and proxy ratings of postinjury behavior, as assessed with the $\operatorname{NBAP}(r=.42 ; p<$ $.05)$ and the DEX $(r=.48 ; p<.05)$. In contrast, in the control group, self ratings and proxy ratings of either premorbid or postinjury behavior did not correlate $(p>.08)$. Within the control group, proxy ratings of premorbid behavior correlated with proxy ratings of postinjury behavior on all three questionnaires $(r>.65 ; p<.01)$. However, within the patient group, there was no significant correlation between proxy ratings of premorbid and postinjury as assessed with the NBAP $(p=.24)$, the KAS-R $(p=.35)$, or the DEX $(p=.06)$. Premorbid behavior was a predictor of postinjury behavior in the control group but not in the patient group. Finally, proxy 
ratings of postinjury behavior on all three questionnaires were associated with TBI severity, as represented by GCS score $(r \geq-.37 ; p<.05)$. Patients who had suffered more severe head injuries, that is, lower GCS scores, displayed more behavioral problems at follow-up.

\section{Relationship Between Test Performance and Postinjury Behavior}

Emotion recognition, understanding intentions ("theory of mind") and cognitive flexibility were each assessed with two independent tests. Because all tests were scored on different scales, performance was expressed as $z$-scores based on the means and standard deviations of the control group. Figure 1 displays the results of the TBI group at the first assessment and at 1-year follow-up. Comparison of the patients' mean $z$-scores against the mean of the controls $(M=0 ; S D=1)$ revealed significantly impaired performance at the first and second assessment on all tests $(p<$ $.03)$, except on the Brixton test at initial assessment where the group difference was no more than a trend $(p=.08)$. The next step was to examine the association between these test scores and postinjury behavior.

Correlations between proxy ratings of postinjury behavior and test performance in the TBI group shortly after injury and at 1-year follow-up are presented in Table 2. To reduce the number of correlations, composite scores were formed by summing the $z$-scores of the two tests assessing emotion recognition, ToM, or cognitive flexibility. Composite scores showed significant associations between emotion recognition, ToM, and cognitive flexibility at initial assessment and follow-up. In addition, proxy ratings of postinjury behavior on the three questionnaires correlated highly. However, there were no significant correlations between test performance at initial assessment or at follow-up and proxy ratings of postinjury behavior in the patients.

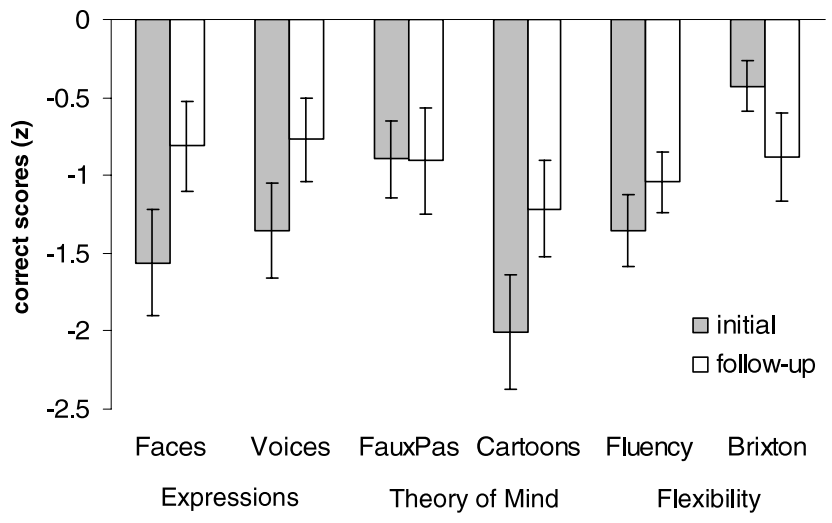

Fig. 1. Performance of the traumatic brain injury group at initial assessment and 12-month follow-up on tests of theory of mind, flexibility, and expression recognition. Performance is expressed as average $z$-scores based on means and standard deviations of the control group. Controls' mean is $0(S D=1)$. Error bars represent standard errors.
Table 2. Correlations between proxy ratings of postinjury behavior in the TBI group and test composite scores at initial assessment (A) and at 1-year follow-up (B)

\begin{tabular}{|c|c|c|c|c|c|}
\hline \multicolumn{6}{|l|}{ A } \\
\hline & Expressions & Flexibility & KAS-R & DEX & NBAP \\
\hline ToM & $0.54 *$ & $0.62 * *$ & 0.10 & 0.06 & 0.07 \\
\hline Expressions & & $0.67 * *$ & 0.05 & 0.07 & 0.08 \\
\hline Flexibility & & & 0.23 & 0.16 & 0.20 \\
\hline KAS-R & & & & $0.88^{* *}$ & $0.87 * *$ \\
\hline DEX & & & & & $0.84 * *$ \\
\hline \multicolumn{6}{|l|}{ B } \\
\hline & Expressions & Flexibility & KAS-R & DEX & NBAP \\
\hline ToM & $0.62 * *$ & $0.78 * *$ & 0.08 & 0.01 & 0.03 \\
\hline Expressions & & $0.57 *$ & 0.11 & -0.14 & 0.01 \\
\hline Flexibility & & & 0.23 & 0.12 & 0.23 \\
\hline KAS-R & & & & $0.88^{* *}$ & $0.87 * *$ \\
\hline DEX & & $0.84 * *$ & & & \\
\hline
\end{tabular}

Note . $\mathrm{TBI}=$ traumatic brain injury; NBAP $=$ Neuropsychology Behavior and Affect Profile; DEX $=$ Dysexecutive Questionnaire; KAS-R = Katz Adjustment ScaleRevised; ToM $=$ Theory of Mind.

$* p<.01$.

$* * p<.001$

Associations between self ratings of postinjury behavior within the TBI group, as assessed on the DEX and NBAP, and test performance were comparable to the results based on proxy ratings, in that none of the test scores at initial assessment or at follow-up correlated with self ratings of postinjury behavior $(r \leq .30 ; p>.10)$.

Finally, we investigated correlations between test performance and postinjury ratings on subscales of the NBAP, DEX, and KAS-R. These correlations, calculated on proxy and self ratings, when available, were very similar to those based on the questionnaires' total scores; none of the subscales were significant correlated with test performance shortly after injury or at follow-up. The only exception was a negative correlation $(r=-.41 ; p<.05)$ between the emotion recognition composite score at follow-up and NBAP Pragnosia (pragmatics of communication) proxy ratings; patients whose recognition of emotional expressions was poor were rated as showing less effective communication.

\section{DISCUSSION}

The aim of this study was to investigate whether deficits in emotion recognition, understanding intentions (ToM), or cognitive flexibility might underlie changes in social behavior following TBI. If this were the case, we would expect poorer test performance to be associated with more severe behavioral problems. Compared with orthopedic controls, the TBI group proved impaired on expression recognition, ToM, and cognitive flexibility soon after injury and at 1-year followup. Proxy ratings of behavior showed an increase in behavioral problems 1 year following TBI, but not following orthopedic injury. However, there was no association between test performance, shortly after injury or at follow-up, and postinjury behavior in the TBI group. The only correlation to reach significance was between emotion recognition at follow-up 
and the NBAP Pragnosia subscore. Although reminiscent of observations by Hornak et al. (1996) and Pettersen (1991) of less appropriate social communication among those patients with TBI who had difficulties recognizing emotions, the correlation was not strong and had not reached significance if the $\alpha$ level had been corrected for multiple comparisons.

It seems unlikely that the absence of significant associations between test performance and behavioral ratings was due to poor validity of the questionnaires used. Each of the three questionnaires had previously been used in TBI samples (e.g., Goran \& Fabiano, 1993; Hanks et al., 1999; Hart et al., 2005; Mathias \& Coats, 1999), and we found strong correlations between ratings on the KAS-R, DEX, and NBAP. Consistent with previous work, ratings of postinjury behavior were associated with TBI severity (Kendall \& Terry, 1996; Tate \& Broe, 1999) and with relatives' distress (Kinsella et al., 1991; Koskinen, 1998; Marsh et al., 2002).

The tests to assess emotion recognition, ToM, and cognitive flexibility have all been used in previous patient studies and proved sensitive enough to reveal impairments in the current TBI group. Still, it is possible that alternative tests would have resulted in stronger associations with postinjury behavior. Associations between executive functioning and psychosocial outcome following TBI have been found with the Wisconsin Card Sorting Test (WCST: Vilkki et al., 1994), Trail Making Test (Nybo et al., 2004), Fluency tests (Ownsworth \& Fleming, 2005; Tate, 1999), Tinker Toy Test (Martzke et al., 1991) and a Go-Nogo Task (Bogod et al., 2003). However, in other studies the WCST (Martzke et al., 1991; Mathias \& Coats, 1999; Tate, 1999), Fluency tests (Milders et al., 2003; Vilkki et al., 1994) and Tinker Toy Test (Ownsworth \& Fleming, 2005) failed to predict psychosocial outcome, and similar null results were found with the Stroop Test (Bogod et al., 2003; Vilkki et al., 1994), the Key Search Test (Ownsworth \& Fleming, 2005; Wood \& Liossi, 2006), and the Brixton Test (Wood \& Liossi, 2006). These conflicting findings illustrate the difficulty of measuring executive functioning (Crawford \& Henry, 2005), and specifically those aspects relevant for psychosocial outcome. In the current study, we focused on the relationship between cognitive flexibility and postinjury behavior. Future studies could investigate more systematically the contribution of different aspects of executive functioning toward predicting emotional and social behavior following TBI.

The variety in available ToM tests is limited compared with the many executive function tests, but as with executive functioning the same ToM test could be associated with ratings of behavior in one study but not another. Gregory et al. (2002) and Milders et al. (2003) found the Faux Pas to correlate with social and emotion behavior, whereas no such correlation was found in the current study. Emotion recognition is typically assessed by asking patients to recognize pictures of facial expressions. Croker and McDonald (2005) and Hornak et al. (1996) found associations between expression recognition and subjective emotional experience in TBI patients; however, neither study reported an association between expression recognition and ratings of behavior.
Hooker and Park (2002) reported associations between emotion recognition and social behavior in patients with schizophrenia, but only in three of the nine domains of social functioning tested and one correlation was no more than a trend.

A limitation of the present study was its sample size. A larger sample may have resulted in more significant correlations. This argument could especially apply to the correlations between cognitive flexibility and behavior, which were comparatively high at around 0.2 . However, as discussed above, evidence for a relationship between impairments in executive functioning and social behavior following TBI is ambiguous. In addition, our sample size was similar that in studies by Nybo et al. $(2004 ; n=33)$ or Tate (1999; $n=30$ ) who did find associations between executive function and postinjury behavior. It is unlikely that a larger sample would have revealed significant associations between behavior and emotion recognition or ToM, as most of these correlations were very low $(r<.10)$.

Through consecutive recruitment we hoped to obtain a representative sample of patients with TBI, but consecutive recruitment also resulted in a wide variation in TBI severity. This variation may have been beneficial for the chances to find correlations between tests and behavior, but because patients were not selected, only a limited number of patients may actually have experienced serious changes in behavior, thus limiting the possibilities to identify shared deficits in these patients. Future studies may select only patients with severe TBI, who are more likely to develop behavioral problems (Kendall \& Terry, 1996; Tate \& Broe, 1999).

Despite the limitations of the present study our findings give little reason to conclude that impairments in emotion recognition or ToM were related to changes in social behavior. While numerous studies into deficits in emotion recognition or ToM in patients with brain damage or psychiatric disorders referred to the implications of such impairments for social functioning, the proposed association still has to be established. The general impression conveyed by the small number of neuropsychological studies, including the current study, that did examine the association between impairment in emotion recognition, ToM, and behavior, is that evidence for such an association is weak. The implication is that to further understand the deficits underlying changes in social behavior following TBI, other deficits may need to be considered. Possible candidates to consider include impairments in the ability to empathize with others, initiating actions, controlling frustration and anger, or interpretation of more subtle, and, therefore, more realistic, expressions of emotions, than were used in the present study (Adolphs et al., 2002; McDonald et al., 2004; Prigatano \& Gray, 2007; Wells et al., 2005).

\section{ACKNOWLEDGMENTS}

We thank staff at the Departments of Neurosurgery and Orthopaedic Trauma Unit (Aberdeen Royal Infirmary) for their cooperation; Clare Scott, Lindsey Campbell, Janine Catto, Rebecca 
Hartmann, Kristina Küper for assistance with the data collection; and Laura Bach and Francesca Happé for the Cartoon test. This research was supported by the Chief Scientist Office of the Scottish Executive $(\mathrm{CZH} / 4 / 14)$.

\section{REFERENCES}

Adolphs, R., Baron-Cohen, S., \& Tranel, D. (2002). Impaired recognition of social emotions following amygdala damage. Journal of Cognitive Neuroscience, 14, 1264-1274.

Bibby, H. \& McDonald, S. (2005). Theory of mind after traumatic brain injury. Neuropsychologia, 43, 99-114.

Bogod, N., Mateer, C., \& MacDonald, S. (2003). A comparison of measures and their relationship to executive functions. Journal of the International Neuropsychological Society, 9, 450-458.

Bowers, D., Blonder, X.L., \& Heilman, K.M. (1998). Florida Affect Battery. Gainesville, FL: University of Florida, Center for Neuropsychological Studies.

Bowman, M. (1996). Ecological validity of neuropsychological and other predictors following head injury. The Clinical Neuropsychologist, 10, 382-396.

Braun, C., Baribeau, J., Ethier, M., Daigneault, S., \& Proulx, R. (1989). Processing of pragmatic and affective information by patients with closed-head injuries. Brain Injury, 3, 5-17.

Brooks, N., Campsie, L., Symington, C., Beattie, A., \& McKinlay, W. (1986). The five year outcome of severe blunt head injury: A relative's view. Journal of Neurology, Neurosurgery, and Psychiatry, 49, 764-770.

Brooks, N., McKinlay, W., Symington, C., Beattie, A., \& Campsie, L. (1987). Return to work within the first seven years of severe head injury. Brain Injury, 1, 5-19.

Burgess, P. \& Shallice, T. (1997). Hayling and Brixton Tests. London: Harcourt Assessment.

Channon, S. \& Crawford, S. (2000). The effects of anterior lesions on performance on a story comprehension test: Left anterior impairment on a theory of mind-type task. Neuropsychologia, 38, 1006-1017.

Channon, S., Pellijeff, A., \& Rule, A. (2005). Social cognition after head injury: Sarcasm and theory of mind. Brain and Language, 93, 123-134.

Cicerone, K. \& Tanenbaum, L. (1997). Disturbances of social cognition after traumatic orbitofrontal brain injury. Archives of Clinical Neuropsychology, 12, 173-188.

Corrigan, P. (1997). The social perceptual deficits of schizophrenia. Psychiatry, 60, 309-326.

Crawford, J.R. \& Henry, J.D. (2005). Assessment of executive deficits. In P.W. Halligan \& N. Wade (Eds.), The Effectiveness of Rehabilitation for Cognitive Deficits (pp. 233-245). London: Oxford University Press.

Croker, V. \& McDonald, S. (2005). Recognition of emotion from facial expression following traumatic brain injury. Brain Injury, 19, 787-799.

Downes, J., Sharp, H., Costall, B., Sagar, H., \& Howe, J. (1993). Alternating fluency in Parkinson's disease. An evaluation of the attentional control theory of cognitive impairment. Brain, 116, 887-902.

Ekman, P. \& Friesen, W. (1976). Pictures of Facial Affect. Palo Alto, CA: Consulting Psychologists Press.

Godfrey, H. \& Shum, D. (2000). Executive functioning and the application of social skills following traumatic brain injury. Aphasiology, 14, 433-444.
Goodglass, H. \& Kaplan, E. (1983). Boston Diagnostic Aphasia Examination. Media, PA: Williams \& Wilkins.

Goran, D. \& Fabiano, R. (1993). The scaling of the Katz Adjustment Scale in a traumatic brain injury rehabilitation sample. Brain Injury, 7, 219-229.

Green, R., Turner, G., \& Thompson, W. (2004). Deficits in facial emotion perception in adults with recent traumatic brain injury. Neuropsychologia, 42, 133-141.

Gregory, C., Lough, S., Stone, V., Erzinclioglu, S., Martin, L., Baron-Cohen, S., \& Hodges, J. (2002). Theory of mind in patients with frontal variant frontotemporal dementia and Alzheimer's disease: Theoretical and practical implication. Brain, $125,752-764$.

Hanks, R., Temkin, N., Machamer, J., \& Dikmen, S. (1999). Emotional and behavioral adjustment after traumatic brain injury. Archives of Physical Medicine and Rehabilitation, 80, 991-999.

Happé, F., Brownell, H., \& Winner, E. (1999). Acquired 'theory of mind' impairments following stroke. Cognition, 70, 211-240.

Happé, F., Malhi, G., \& Checkley, S. (2001). Acquired mindblindness following frontal lobe surgery? A single case study of impaired 'theory of mind' in a patient treated with stereotactic anterior capsulotomy. Neuropsychologia, 39, 83-90.

Hart, T., Whyte, J., Kim, J., \& Vaccaro, M. (2005). Executive function and self-awareness of "real-world" behavior and attention deficits following traumatic brain injury. Journal of Head Trauma Rehabilitation, 20, 333-347.

Hooker, C. \& Park, S. (2002). Emotion processing and its relationship to social functioning in schizophrenia patients. Psychiatry Research, 112, 41-50.

Hopkins, M., Dywan, J., \& Segalowitz, S. (2002). Altered electrodermal response to facial expression after closed head injury. Brain Injury, 16, 245-257.

Hornak, J., Rolls, E.T., \& Wade, D. (1996). Face and voice expressions identification in patients with emotional and behavioural changes following ventral lobe damage. Neuropsychologia, 34 , 247-261.

Jackson, H. \& Moffat, N. (1987). Impaired emotional recognition following severe head injury. Cortex, 23, 293-300.

Kendall, E. \& Terry, D. (1996). Psychosocial adjustment following closed head injury: A model for understanding individual differences and predicting outcome. Neuropsychological Rehabilitation, 6, 101-132.

Kinsella, G., Packer, S., \& Olver, J. (1991). Maternal reporting of behaviour following very severe blunt head injury. Journal of Neurology, Neurosurgery, and Psychiatry, 54, 422-426.

Koskinen, S. (1998). Quality of life 10 years after a very severe traumatic brain injury (TBI): The perspective of the injured and the closest relative. Brain Injury, 12, 631-648.

Levin, H.S. (1995). Neurobehavioral outcome of closed head injury: Implications for clinical trials. Journal of Neurotrauma, 12, 601-610.

Malia, K., Powell, G., \& Torode, S. (1995). Personality and psychosocial function after brain injury. Brain Injury, 9, 697-712.

Marsh, N., Kersel, D., Havill, J., \& Sleigh, J. (2002). Caregiver burden during the year following severe traumatic brain injury. Journal of Clinical and Experimental Neuropsychology, 24, 434-447.

Martzke, J., Swan, C., \& Varney, N. (1991). Posttraumatic anosmia and orbital frontal damage: Neuropsychological and neuropsychiatric correlates. Neuropsychology, 5, 213-225.

Mathias, J.L. \& Coats, J.L. (1999). Emotional and cognitive sequelae to mild traumatic brain injury. Journal of Clinical and Experimental Neuropsychology, 21, 200-215. 
McDonald, S. \& Flanagan, S. (2004). Social perception deficits after traumatic brain injury: Interaction between emotion recognition, mentalizing ability and social communication. Neuropsychology, 18, 572-579.

McDonald, S., Flanagan, S., Martin, I., \& Saunders, C. (2004). The ecological validity of the TASIT: A test of social perception. Neuropsychological Rehabilitation, 14, 285-302.

McDonald, S. \& Saunders, J. (2005). Differential impairment in recognition of emotion across different media in people with severe traumatic brain injury. Journal of the International Neuropsychological Society, 11, 392-399.

Milders, M., Fuchs, S., \& Crawford, J.R. (2003). Neuropsychological impairments and changes in emotional and social behaviour following severe traumatic brain injury. Journal of Clinical and Experimental Neuropsychology, 25, 157-172.

Milders, M., Ietswaart, M., Crawford, J.R., \& Currie, D. (2006). Impairments in 'theory of mind' shortly after traumatic brain injury and at one-year follow-up. Neuropsychology, 20, 400-408.

Morton, M. \& Wehman, P. (1995). Psychosocial and emotional sequelae of individuals with traumatic brain injury: A literature review and recommendations. Brain Injury, 9, 81-92.

Nelson, L., Drebing, C., Satz, P., \& Uchiyama, C.L. (1998). Personality change in head trauma: A validity study of the Neuropsychology Behavior and Affect Profile. Archives of Clinical Neuropsychology, 13, 549-560.

Nybo, T., Sainio, M., \& Müller, K. (2004). Stability of vocational outcome in adulthood after moderate to severe preschool brain injury. Journal of the International Neuropsychological Society, 10, 719-723.

Office for National Statistics. (2005). The National Statistics Socioeconomic Classification: User Manual. Basingstoke, UK: Palgrave Macmillan.

Ownsworth, T. \& Fleming, J. (2005). The relative importance of metacognitive skills, emotional status, and executive function in psychosocial adjustment following acquired brain injury. Journal of Head Trauma Rehabilitation, 20, 315-332.

Ownsworth, T. \& McKenna, K. (2004). Investigation of factors related to employment outcome following traumatic brain injury: A critical review and conceptual model. Disability and Rehabilitation, 26, 765-784.

Pettersen, L. (1991). Sensitivity to emotional cues and social behavior in children and adolescents after head injury. Perception and Motor Skills, 73, 1139-1150.
Prigatano, G. (1992). Personality disturbances associated with traumatic brain injury. Journal of Consulting and Clinical Psychology, 60, 360-368.

Prigatano, G. (1999). Principles of Neuropsychological Rehabilitation. New York: Oxford University Press.

Prigatano, G. \& Gray, J. (2007). Parental concerns and distress after pediatric traumatic brain injury: A qualitative study. Brain Injury, 21, 721-729.

Spell, L. \& Frank, E. (2000). Recognition of nonverbal communication of affect following traumatic brain injury. Journal of Nonverbal Behavior, 24, 285-300.

Stone, V., Baron-Cohen, S., \& Knight, R. (1998). Frontal lobe contributions to theory of mind. Journal of Cognitive Neuroscience, 10, 640-656.

Tate, R.L. (1999). Executive dysfunction and characterological changes after traumatic brain injury: Two sides of the same coin? Cortex, 35, 39-55.

Tate, R.L. \& Broe, G.A. (1999). Psychosocial adjustment after traumatic brain injury: What are the important variables. Psychological Medicine, 29, 713-725.

Teasdale, G. \& Jennett, B. (1974). Assessment of coma and impaired consciousness. Lancet, 2, 81-84.

Vilkki, J., Ahola, K., Holst, P., Ohman, J., Servo, A., \& Heiskanen, O. (1994). Prediction of psychosocial recovery after head injury with cognitive test and neurobehavioral ratings. Journal of Clinical and Experimental Neuropsychology, 16, 325-338.

Wells, R., Dywan, J., \& Dumas, J. (2005). Life satisfaction and distress in family caregivers as related to specific behavioural changes after traumatic brain injury. Brain Injury, 19, $1105-1115$.

Wilson, B., Alderman, N., Burgess, P., Emslie, H., \& Evans, J. (1996). Behavioural Assessment of the Dysexecutive Syndrome. Bury St. Edmunds, UK: Thames Valley Test Company.

Wood, R. \& Liossi, C. (2006). The ecological validity of executive test in a severely brain injured sample. Archives of Clinical Neuropsychology, 21, 429-437.

Zigmond, A. \& Snaith, R. (1983). The Hospital Anxiety and Depression Scale. Acta Psychiatrica Scandinavica, 67, 361-370. 